\title{
Reduced-Thickness-Dependent Polarized Light Transmittance of Magnetic Fluids under Longitudinal Magnetic Field
}

\author{
Shengli Pu, Xuekun Bai, and Lunwei Wang \\ College of Science, University of Shanghai for Science and Technology, Shanghai 200093, China \\ Correspondence should be addressed to Shengli Pu, shlpu@usst.edu.cn
}

Received 4 May 2011; Revised 5 June 2011; Accepted 5 July 2011

Academic Editor: Mikhail Noginov

Copyright () 2011 Shengli Pu et al. This is an open access article distributed under the Creative Commons Attribution License, which permits unrestricted use, distribution, and reproduction in any medium, provided the original work is properly cited.

The polarized light transmittance of magnetic fluids under longitudinal magnetic field (parallels the propagation direction of the incident light) is simulated theoretically. The investigated samples are with different reduced thickness $(d / \lambda)$ in a wide range. Theoretical simulations reveal that the sample reduced thickness has a significant influence on the polarized light transmittance. The thin and thick samples have distinctly different dependence of polarized light transmittance on magnetic field strength. Based on the reduced-thickness- and magnetic-field-dependent polarized light transmittance, several magnetic-fluid-based photonic devices are proposed and discussed.

\section{Introduction}

Magnetic fluids as optical functional materials have attracted much attention recently. The promising prospect in optical applications and photonic devices is disclosed through versatile experimental results in the laboratory. For example, Ge et al. have experimentally realized the magnetic-fieldresponsive photonic crystals by magnetically induced colloidal assembly within magnetic fluids [1-3]. Zhang et al. have observed the magnetic field modulation of light transmission through binary magnetic fluid films [4]. The magnetic-field-dependent light transmittance is one of the important optical properties of magnetic fluids, which is usually employed to design some photonic devices and should be investigated in depth. This work will focus on the transmittance of polarized light passing through magnetic fluids under longitudinal magnetic field.

The longitudinal magnetic field means that the magnetic field parallels the propagation direction of the incident light. For the longitudinal magnetic field configuration, the magnetic-field-dependent optical transmittance of magnetic fluids is chiefly assigned to the column/chain formation of magnetic particles along the magnetic field direction and the related geometric shadowing effect [5-8]. If the polarized light is applied for the longitudinal magnetic field configuration, Faraday rotation and geometric shadowing effect will happen simultaneously. In our previous work, we have disclosed that several parameters may influence the magnetic-field-dependent optical transmittance of magnetic fluids due to hybrid effects of Faraday rotation and geometric shadowing [9]. But only the thin samples with small values of reduced thickness $(d / \lambda$, where $d$ is the thickness of the magnetic fluids and $\lambda$ is the wavelength of the incident light in vacuum) have been considered in that work. In this work, the magnetic-field-dependent optical transmittance of magnetic fluids samples with reduced thickness in a wide range has been investigated. The results in this work may be helpful for designing several polarized optical devices based on magnetic fluids and can give guidelines for improving their quality.

\section{Formulism}

For an oil-based ferrite magnetic fluid with saturation magnetization of $100 \mathrm{Oe}$, viscosity of $9 \mathrm{mPa} \cdot \mathrm{s}$, and average particle diameter of $10 \mathrm{~nm}$, the longitudinal magnetic-fieldinduced transmitted power of polarized light due to hybrid effects of Faraday rotation and geometric shadowing is given by (the polarization direction of the analyzer is set $45^{\circ}$ with 
respect to that of the incident light to obtain the maximum sensitivity) [9]

$$
\begin{aligned}
& \text { P } \\
& =\left[76.58245-17.00193 \exp \left(-1.04473 \times 10^{-5} H^{2}\right)\right] \times t \\
& \times \cos ^{2}\left\{45^{\circ}\right. \\
& \left.-\frac{2 \pi(d / \lambda) \varepsilon_{x y} \varphi_{s}^{\prime} L(\xi) L\left(\xi^{\prime}\right)}{(1+Q)^{2} \sqrt{\varepsilon_{\text {liquid }}} \sqrt{1+\varphi_{s}^{\prime} L(\xi) D\left[1+Q L\left(\xi^{\prime}\right) / \xi^{\prime}\right]}}\right\},
\end{aligned}
$$

where $H$ is the externally magnetic field strength, $t$ is the transmittivity of the analyzer, $\varepsilon_{x y}$ is the off-diagonal term of the dielectric tensor of the magnetic fluids, $Q=\varepsilon_{\text {solid }} / \varepsilon_{\text {liquid }}$ and $D=\left(\varepsilon_{\text {solid }}-\varepsilon_{\text {liquid }}\right) /\left(\varepsilon_{\text {solid }}+\varepsilon_{\text {liquid }}\right) . \varepsilon_{\text {solid }}$ and $\varepsilon_{\text {liquid }}$ are the dielectric constants of the particle aggregation and the remanent liquid phase within the magnetic fluids, respectively. $L(\xi)=\operatorname{coth}(\xi)-1 / \xi$ and $L\left(\xi^{\prime}\right)=\operatorname{coth}\left(\xi^{\prime}\right)-1 / \xi^{\prime}$. $\xi=\mu_{0} V_{0} M_{d} H /(k T)$ and $\xi^{\prime}=\mu_{0}\left(\beta N_{0}\right)_{s} V_{0} M_{d} L(\xi)(H+$ $\left.H_{w}\right) /(k T) . \mu_{0}$ is the permeability in vacuum. $M_{d}$ is the saturation magnetization of the magnetic nanoparticles. $\beta$ is a constant of the order of unity $(\beta<1)[10], N_{0}$ is the number of magnetic nanoparticles in the particle aggregation, $V_{0}(=$ $4 \pi r^{3} / 3$ ) and $r$ are the volume and radius of the single magnetic nanoparticle, respectively. $H_{w}$ is the Weiss internal field representing the interparticle interactions. $k$ is the Boltzmann constant and $T$ is the absolute temperature of the magnetic fluids. $\varphi^{\prime}=\varphi_{s}^{\prime} L(\xi)$ and $\beta N_{0}=\left(\beta N_{0}\right)_{s} L(\xi)$. $\varphi_{s}^{\prime}$ and $\left(\beta N_{0}\right)_{s}$ are the saturated values of $\varphi^{\prime}$ and $\beta N_{0}$, respectively. $\varphi^{\prime}$ is the volume concentration of particle aggregation.

\section{Results and Discussion}

To understand the reduced-thickness-dependent polarized light transmittance of the aforementioned magnetic fluid under longitudinal magnetic field, numerical simulations based on (1) are done. For the calculations, the following reasonable values of the concerned parameters are taken: $\varepsilon_{x y}=0.1[11] ; \varphi_{s}^{\prime}=\phi$, where $\phi=M_{s} / M_{d}$ is the volume fraction of the magnetic nanoparticles within the magnetic fluid and $M_{s}$ is the saturation magnetization of the magnetic fluid; $r=$ $5 \mathrm{~nm}, M_{s}=7.96 \times 10^{3} \mathrm{~A} / \mathrm{m}$ and $M_{d}=4.46 \times 10^{5} \mathrm{~A} / \mathrm{m} \mathrm{[12];}$ $\varepsilon_{\text {solid }}=4.5$ and $\varepsilon_{\text {liquid }}=2.56[13] ;\left(\beta N_{0}\right)_{s} \approx \pi R^{2} d /\left(4 \pi r^{3} / 3\right)=$ $3 \times 10^{8}$ (close to $3 \times 10^{8}$ particles per aggregation), where $R=0.5 \mu \mathrm{m}$ is the radius of the aggregated column [14]. $T=295 \mathrm{~K}$ (room temperature) and $H_{w}=200$ Oe $[13,15]$.

Figure 1 shows the polarized light transmittance with the strength of externally applied magnetic field for magnetic fluid samples with reduced thickness $d / \lambda$ in a wide range. From Figure 1, we can see that the transmitted power increases with magnetic field monotonously for thin magnetic fluid samples $(d / \lambda=3.16,31.6,316$, and 900). This monotonic increase of transmitted power with magnetic field is due to the small Faraday rotation angle $\theta$ for thin samples,

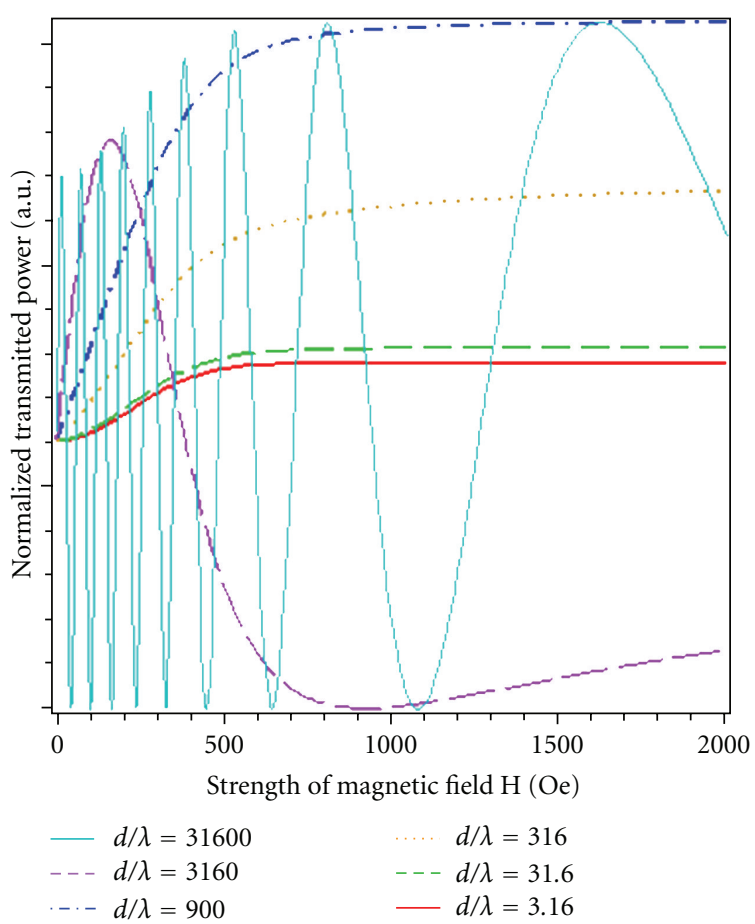

FIGURE 1: The polarized light transmittance as a function of externally applied magnetic field $H$ for magnetic fluid samples with different reduced thickness $d / \lambda$.

where the value of $\theta$ is less than $45^{\circ}$. Numerical calculations indicate that oscillating transmitted power with magnetic field starts to happen when the value of $d / \lambda$ is larger than 900 . Figure 1 depicts this kind of oscillating phenomenon for two thick magnetic fluid samples $(d / \lambda=3160$ and 31600$)$, which demonstrates that strong oscillation occurs for the thicker sample $(d / \lambda=31600)$. The oscillating phenomenon of the transmitted power with magnetic field is assigned to the large Faraday rotation angle $\theta$ for thick samples, where the value of $\theta$ may exceed $45^{\circ}$ (or $90^{\circ}, 135^{\circ}, 180^{\circ}, 225^{\circ}$, etc.). The transmitted power peaks and valleys correspond to Faraday rotation angle $\theta=45^{\circ}+n \cdot 180^{\circ}$ and $\theta=135^{\circ}+n \cdot 180^{\circ}$ $(n=0,1,2,3, \ldots)$, respectively.

To further understand the reduced-thickness-dependent polarized light transmittance for thin samples, Figure 2 plots the polarized light transmittance with the strength of externally applied magnetic field for five thin samples with different reduced thickness $(d / \lambda=150,300,450$, 600 , and 750). Figure 2 shows that the transmitted power increases fast with magnetic field until 500 Oe and tends to saturate beyond 500 Oe. This is assigned to the magneticfield-dependent agglomeration of magnetic nanoparticles within the magnetic fluids. At low field regime, the degree of agglomeration of magnetic nanoparticles within the magnetic fluids increases with the strength of magnetic field. While at high field regime, the agglomeration of magnetic nanoparticles within the magnetic fluids tends to saturate and fewer magnetic nanoparticles will take part in agglomeration with the further augment of magnetic field. 


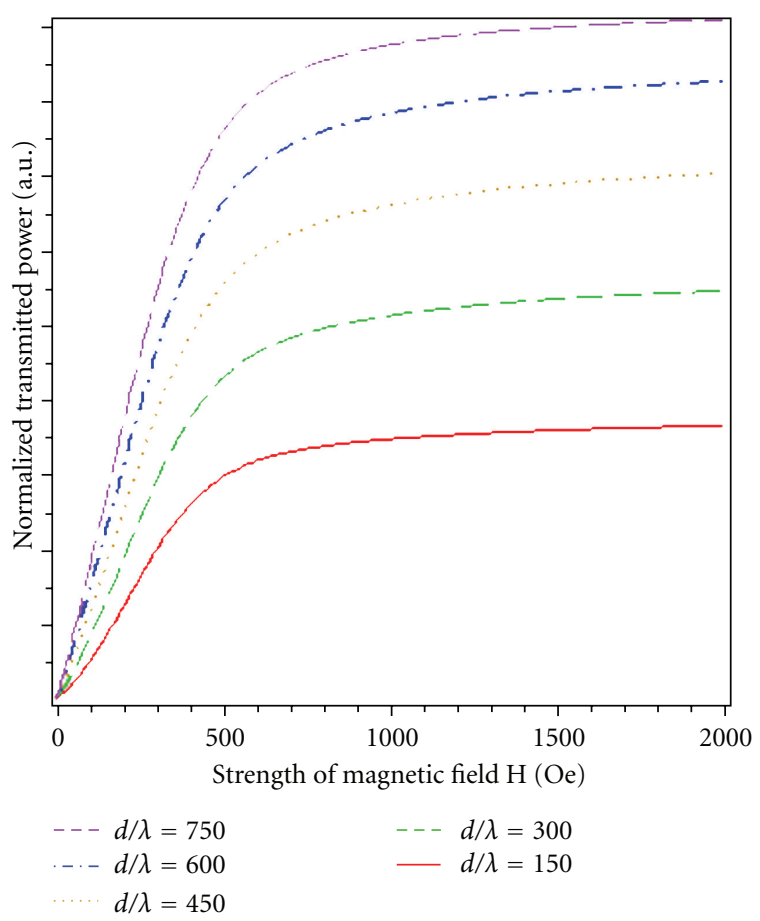

FIgURE 2: The polarized light transmittance as a function of externally applied magnetic field for five thin samples with different reduced thickness $d / \lambda$.

In addition, the sensitivity of the transmitted power with respect to the externally applied magnetic field is high for sample with large value of $d / \lambda$, especially in the low field region. The transmitted power increases with the sample reduced thickness under fixed externally applied magnetic field. The variation of polarized light transmittance with the sample reduced thickness under several fixed magnetic fields $(H=250,500,1000$, and $2000 \mathrm{Oe})$ is extracted from Figure 2 and replotted in Figure 3. From Figure 3, we can see that the transmitted power increases with the sample reduced thickness linearly under fixed magnetic field. For a certain sample (fixed reduced thickness), the transmitted power increases with the strength of the externally applied magnetic field. Besides, the rate of increase of transmitted power with the externally applied magnetic field decreases with the strength of magnetic field.

To further understand the reduced-thickness-dependent polarized light transmittance for thick samples, Figure 4 plots the polarized light transmittance with the strength of externally applied magnetic field for five thick samples with different reduced thickness $(d / \lambda=3160,4160,5160,6160$, and 7160). Figure 4 reveals that the degree of oscillation of the transmitted power with externally applied magnetic field increases with the value of $d / \lambda$, that is, thicker sample have stronger oscillation of the transmitted power with externally applied magnetic field. The strength of magnetic field where the minimum or (first or second) maximum transmitted power takes place decreases with the sample reduced thickness. Moreover, the value of the first or second maximum transmitted power decreases with the sample

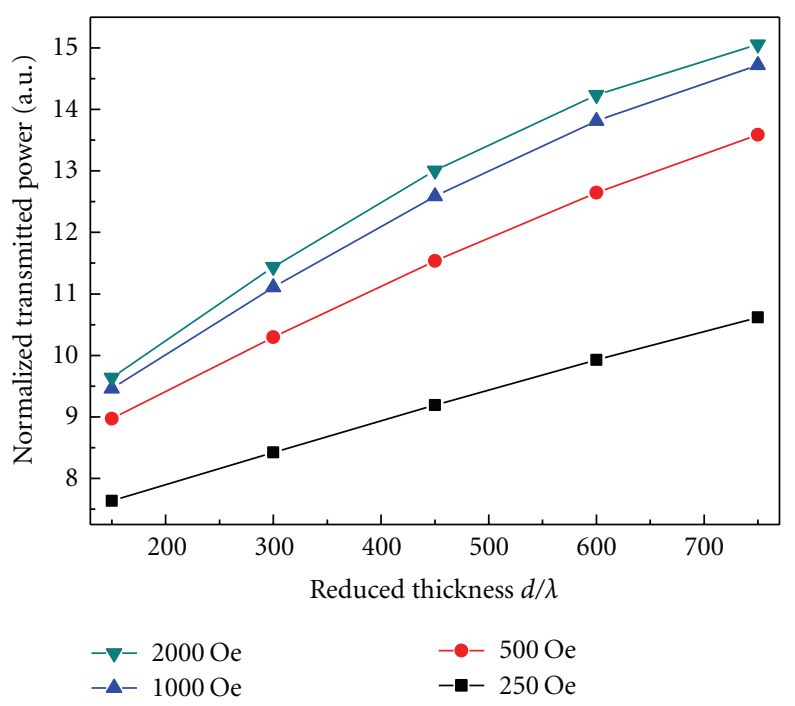

FIGURE 3: The polarized light transmittance as a function of sample reduced thickness $d / \lambda$ under several fixed magnetic fields $H$.

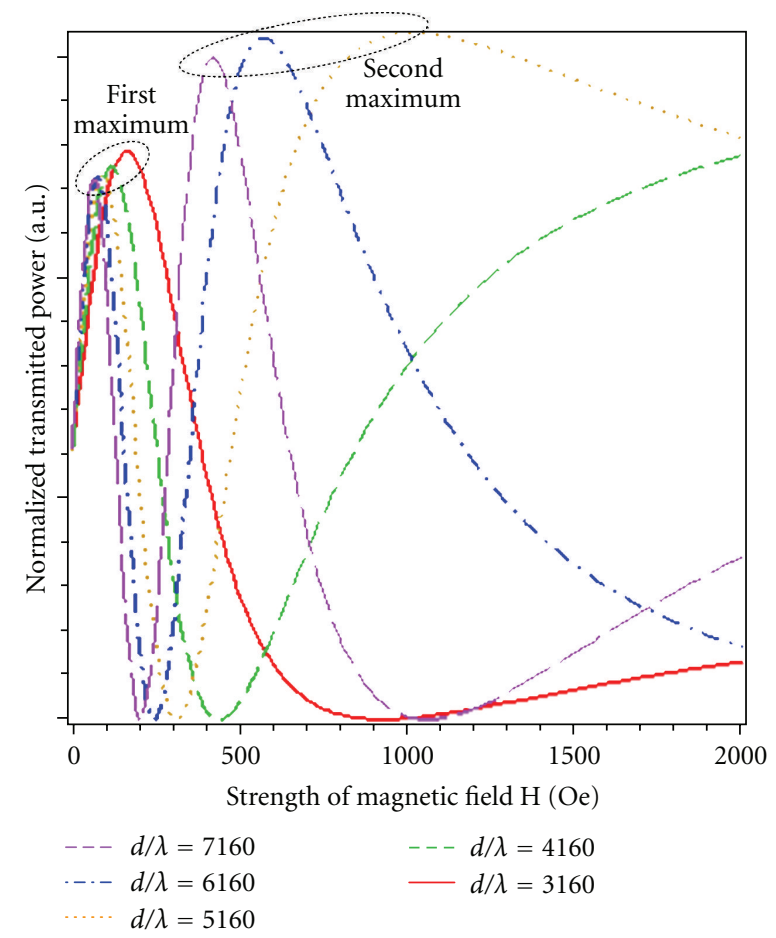

FIGURE 4: The polarized light transmittance as a function of externally applied magnetic field for five thick samples with different reduced thickness $d / \lambda$.

reduced thickness. For a sample with fixed reduced thickness, the value of the second maximum transmitted power is larger than that of the first maximum transmitted power. To further understand the oscillation of transmitted power with externally applied magnetic field, Figures 5 and 6 give the simulation results of transmitted power with respect to the externally applied magnetic field for samples with very large reduced thickness $(d / \lambda=31600,61600,121600$, 


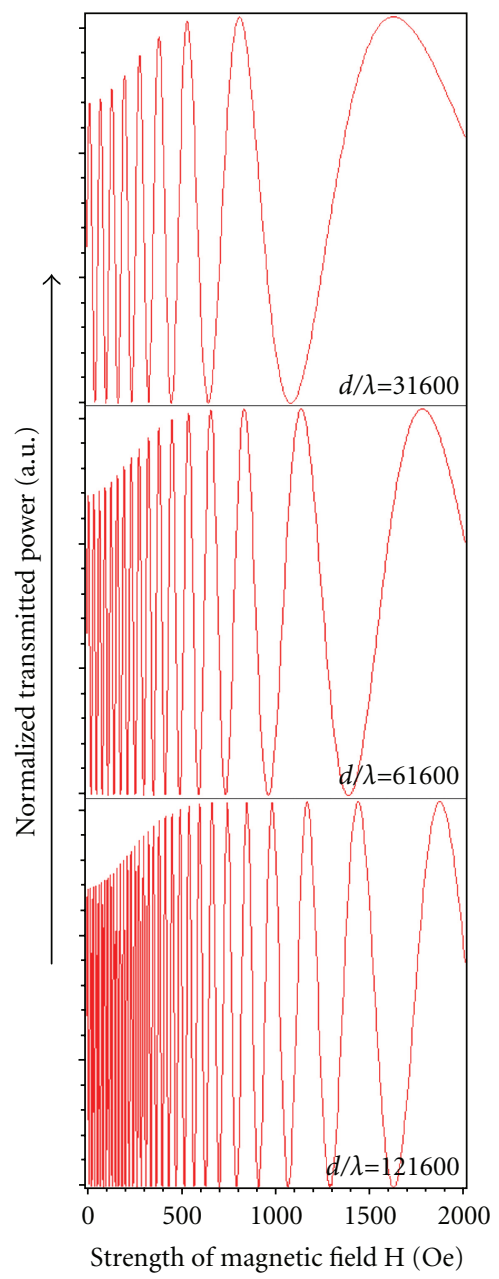

FIGURE 5: The polarized light transmittance as a function of externally applied magnetic field for samples with reduced thickness $d / \lambda=$ 31600, 61600, and 121600 .

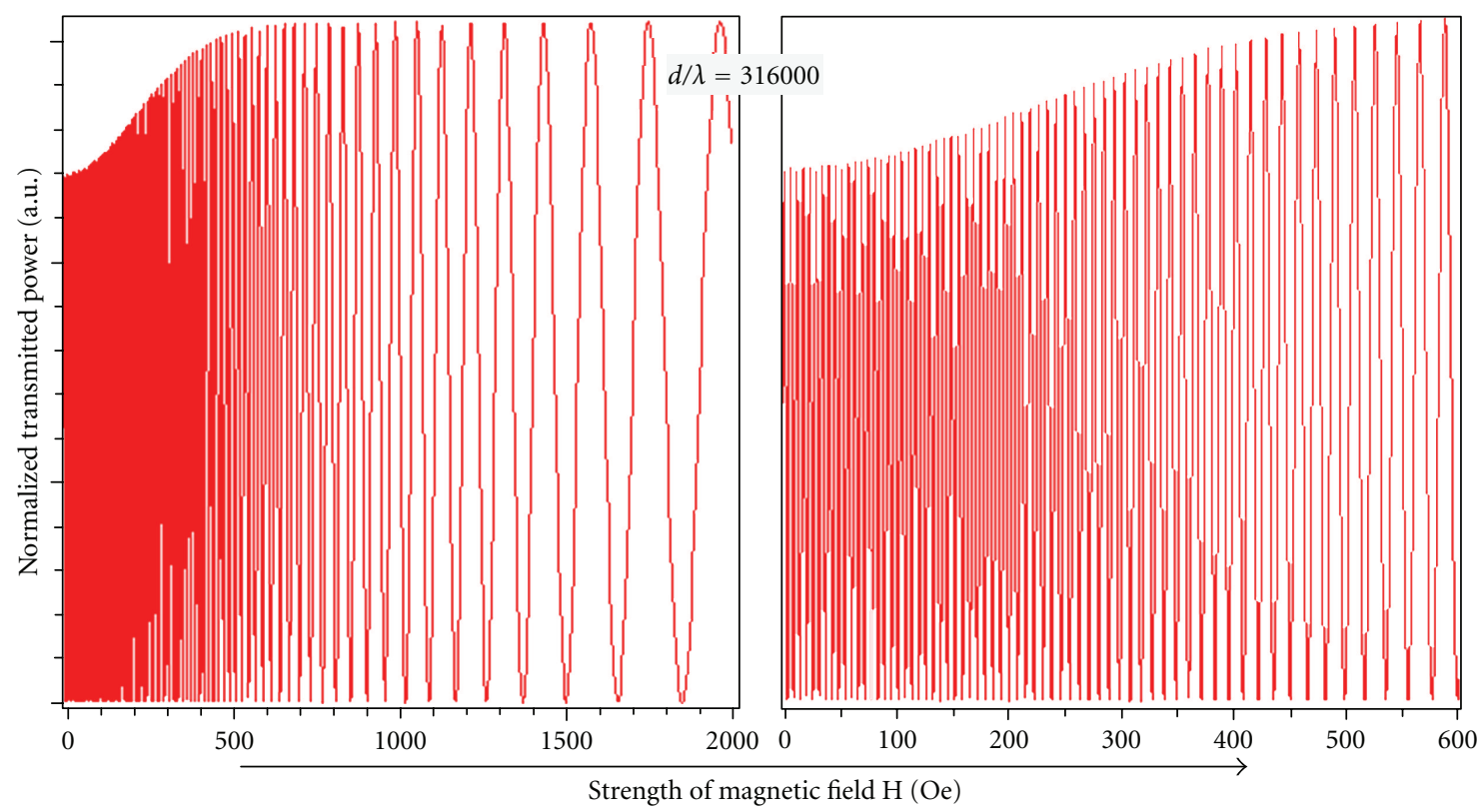

FIGURE 6: The polarized light transmittance as a function of externally applied magnetic field for a sample with reduced thickness $d / \lambda=$ 316000. The right panel is the enlarged plot of the left panel under low magnetic field $(H=0-600 \mathrm{Oe})$. 
and 316000). From Figures 5 and 6, we can clearly see the increasing degree of oscillation of transmitted power with externally applied magnetic field as the sample reduced thickness augments. When the externally applied magnetic field is smaller than $500 \mathrm{Oe}$, the higher the order of the transmitted power peak is, the larger the value of the transmitted power peak will be. While when the externally applied magnetic field is beyond 500 Oe, every transmitted power peak almost has the same value. The distance between the neighboring transmitted power peaks is not constant, but increases when the order of the transmitted power peak grows.

The aforementioned results about the reduced-thicknessdependent polarized light transmittance can be employed to design several magnetic-fluid-based photonic devices operating at longitudinal magnetic field configuration, such as optical attenuator, optical switch, and light modulator. Concretely, the properties of the polarized light transmittance of thin reduced thickness samples are very favorable for designing the tunable optical attenuators. The tuning range and sensitivity of the corresponding attenuators can be adjusted by changing the sample reduced thickness or working magnetic field region. The properties of the polarized light transmittance of thick reduced thickness samples are very favorable for designing light modulator, especially when the working magnetic field is larger than 500 Oe.

\section{Conclusions}

In summary, the transmittance of polarized light passing through magnetic fluid samples with different reduced thickness $(d / \lambda)$ are simulated when considering both the geometric shadowing and Faraday rotation effects. Simulation results show that the polarized light transmittance of thin samples $(d / \lambda<900)$ increase monotonously with magnetic field for certain sample (fixed value of $d / \lambda$ ). They also increase monotonously with sample reduced thickness under fixed magnetic field. The oscillation of transmitted power with regard to magnetic field is observed for thick samples $(d / \lambda>900)$ and the degree of oscillation increases with sample reduced thickness. The results of this work may be helpful for designing some photonic devices or tunable photonic devices, such as optical attenuator, light modulator, and optical switch. The performance of the corresponding photonic devices can be improved by choosing the appropriate parameters, that is, sample reduced thickness and working magnetic field region.

\section{Acknowledgments}

This research is supported by the National Natural Science Foundation of China (no. 10704048) and Innovation Program of Shanghai Municipal Education Commission (no. 11YZ120).

\section{References}

[1] J. Ge and Y. Yin, "Responsive photonic crystals," Angewandte Chemie, vol. 50, no. 7, pp. 1492-1522, 2011.

[2] J. Ge, S. Kwon, and Y. Yin, "Niche applications of magnetically responsive photonic structures," Journal of Materials Chemistry, vol. 20, no. 28, pp. 5777-5784, 2010.

[3] J. Ge, L. He, Y. Hu, and Y. Yin, "Magnetically induced colloidal assembly into field-responsive photonic structures," Nanoscale, vol. 3, no. 1, pp. 177-183, 2011.

[4] T. Z. Zhang, J. Li, H. Miao, Q. M. Zhang, J. Fu, and B. C. Wen, "Enhancement of the field modulation of light transmission through films of binary ferrofluids," Physical Review E, vol. 82, no. 2, Article ID 021403, 2010.

[5] J. Li, Y. Lin, X. Liu et al., "The modulation of coupling in the relaxation behavior of light transmitted through binary ferrofluids," Optics Communications, vol. 283, no. 6, pp. 1182-1187, 2010.

[6] J. Li, Y. Huang, X. D. Liu, Y. Q. Lin, Q. Li, and R. L. Gao, "Coordinated chain motion resulting in intensity variation of light transmitted through ferrofluid film," Physics Letters A, vol. 372, no. 46, pp. 6952-6955, 2008.

[7] J. Li, X. Liu, Y. Lin et al., "Field modulation of light transmission through ferrofluid film," Applied Physics Letters, vol. 91, no. 25, Article ID 253108, 2007.

[8] L. E. Helseth, "Optical sensor for detecting colloidal phase transitions induced by magnetic fields," Journal of Physics D, vol. 42, no. 10, Article ID 105005, 2009.

[9] S. Pu, M. Dai, and G. Sun, "Longitudinal field-induced polarized light transmittance of magnetic fluids," Optics Communications, vol. 283, no. 20, pp. 4012-4016, 2010.

[10] S. Taketomi, "Magnetic fluid's anomalous pseudo-cotton mouton effects about $10^{7}$ times larger than that of nitrobenzene," Japanese Journal of Applied Physics, vol. 22, no. 7, pp. 1137-1143, 1983.

[11] X. Zhang, J. Schoenes, and P. Wachter, "Kerr-effect and dielectric tensor elements of magnetite $\left(\mathrm{Fe}_{3} \mathrm{O}_{4}\right)$ between 0.5 and $4.3 \mathrm{eV}$," Solid State Communications, vol. 39, no. 1, pp. 189$192,1981$.

[12] R. E. Rosensweig, Ferrohydrodynamics, Cambridge University Press, Cambridge, UK, 1985.

[13] S. Taketomi, M. Ukita, M. Mizukami, H. Miyajima, and S. Chikazumi, "Magnetooptical effects of magnetic fluid," Journal of the Physical Society of Japan, vol. 56, no. 9, pp. 33623374, 1987.

[14] S. Y. Yang, H. E. Horng, C. Y. Hong et al., "Control method for the tunable ordered structures in magnetic fluid microstrips," Journal of Applied Physics, vol. 93, no. 6, pp. 3457-3460, 2003.

[15] K. O'Grady, A. Bradbury, S. W. Charles, S. Menear, J. Popplewell, and R. W. Chantrell, "Curie-Weiss behavior in ferrofluids," Journal of Magnetism and Magnetic Materials, vol. 31-34, no. 2, pp. 958-960, 1983. 

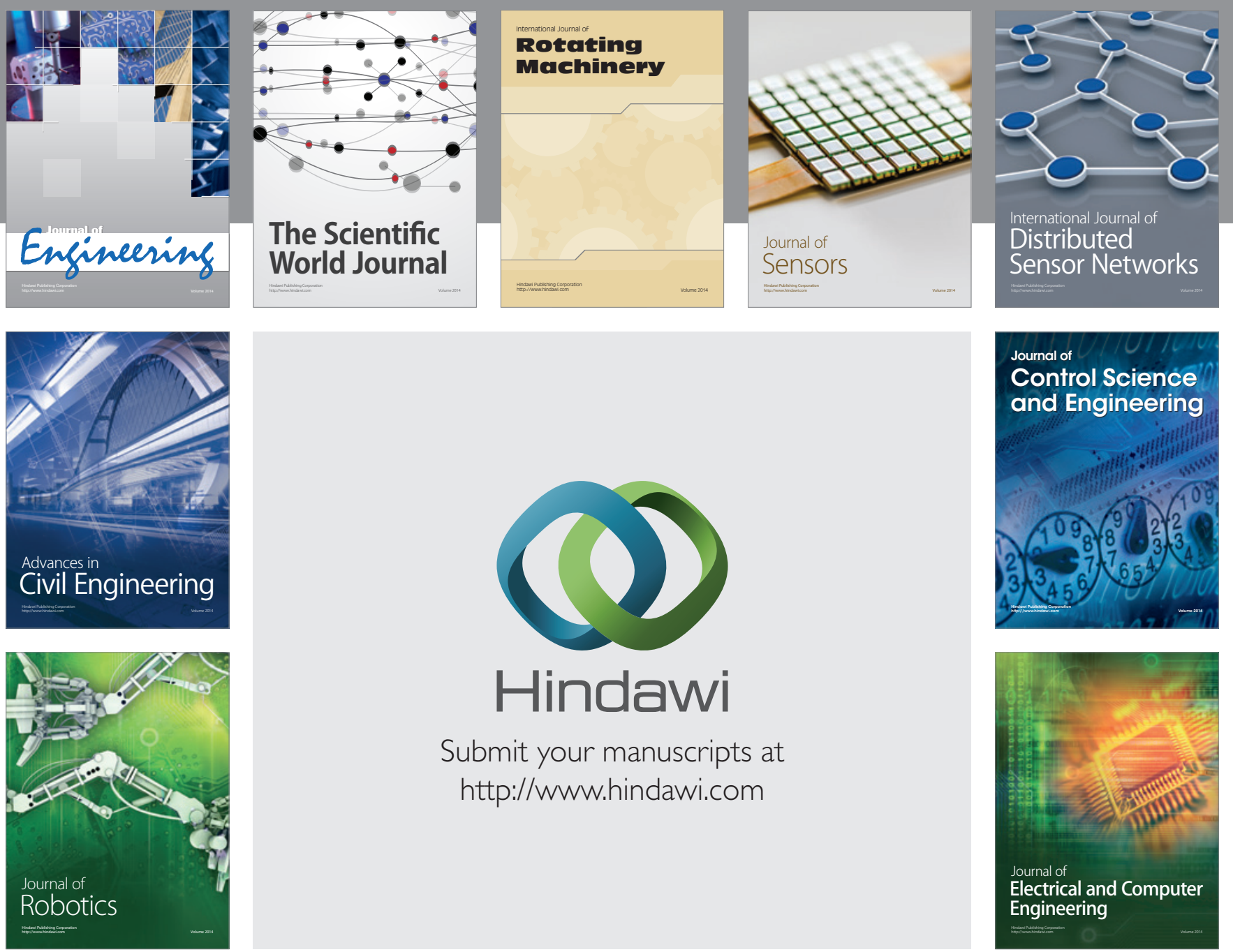

Submit your manuscripts at

http://www.hindawi.com
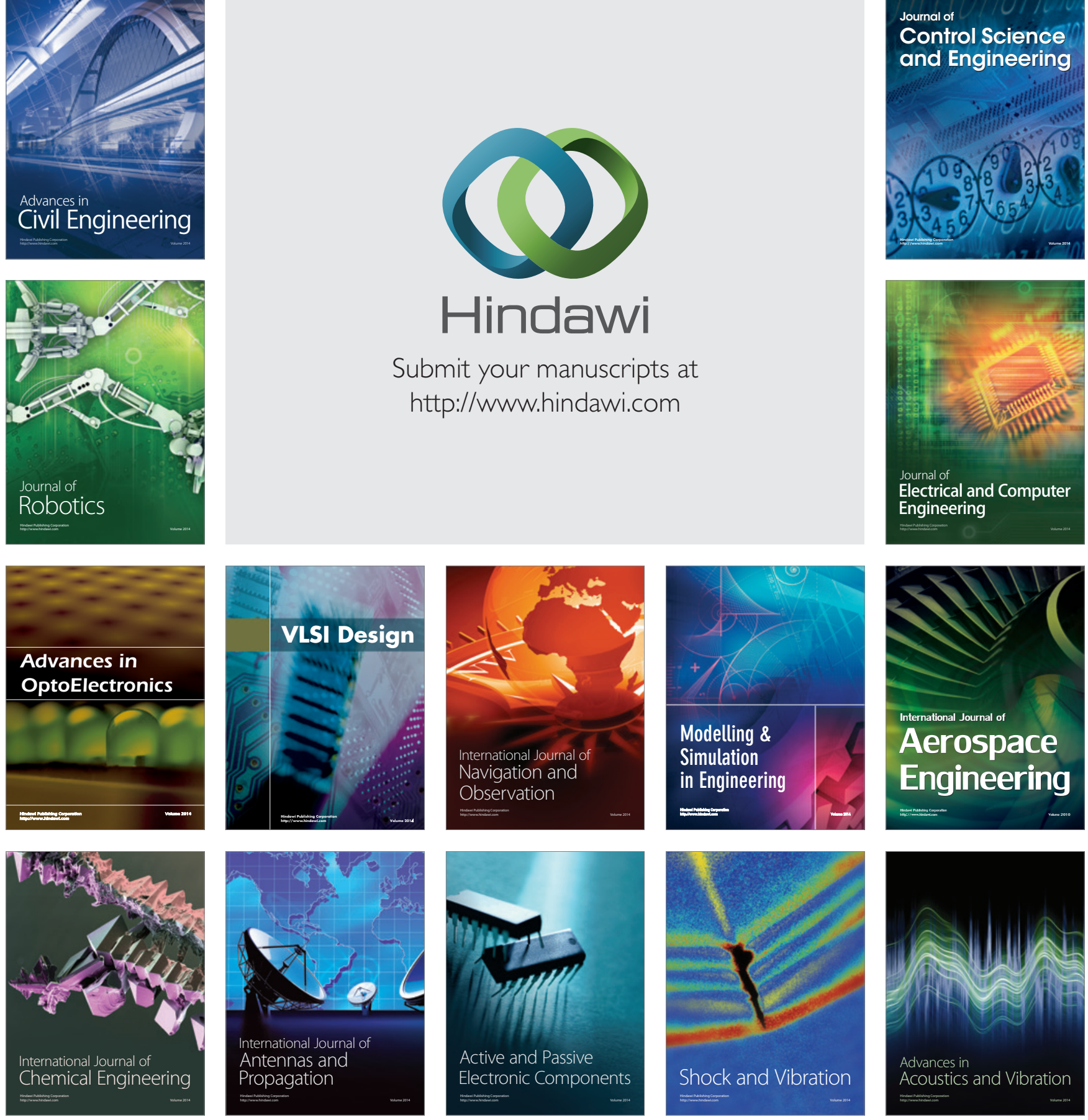